\title{
OS NANOCOSMÉTICOS NO ENVELHECIMENTO FACIAL: revisão de
}

\author{
literatura
}

Andressa Gonçalves Cavalcanti CAMPOS ${ }^{1}$

Leticia Caixeta Ribeiro CUNHA ${ }^{2}$

Rafaela PINHEIRO ${ }^{3}$

Alexandra Azevedo CARVALHO

${ }^{1}$ Pós graduanda em Estética Aplicada - Universidade Vale do Rio Verde - UNINCOR
2Pós graduanda em Estética Aplicada - Universidade Vale do Rio Verde - UNINCOR
${ }^{3}$ Pós graduanda em Estética Aplicada - Universidade Vale do Rio Verde - UNINCOR
${ }^{4}$ Orientadora e Prof ${ }^{\text {a }}$ Ms. do Curso de Graduação e Pós Graduação de Estética e Cosmética/UNINCOR.

Recebido em: 28/01/2015 - Aprovado em: 17/06/2015 - Disponibilizado em: 15/07/2015

Resumo: O envelhecimento é um processo natural, o qual todos estamos suscetíveis. Hoje em dia existem diversas tecnologias que propõem o seu tratamento e consequente amenização de seus sinais cutâneos. Com o aumento de consumo de mercado cosmético, surgem também técnicas inovadoras com finalidades diversas e propostas eficazes. Este presente artigo objetiva revisar a literatura sobre nanocosméticos e sua atuação no envelhecimento. Para estes fins foram feitas diversas buscas nos bancos de dados científicos, tais como Medline, Pubmed, Google Acadêmico. O mercado cosmetológico vem aumentando a cada ano, e os investimentos na área deste seguimento também. A nanotecnologia surgiu como grande inovadora para diversos tratamentos estéticos, trazendo consigo promessas de resultados milagrosos. Cabe aos profissionais desta area conhecer e distinguir tais produtos e suas reais capacidades, malefícios e benefícios. Ainda não se sabe exatamente quais camadas cutâneas são atingidas, se seus efeitos são meramente hidrantes e se os riscos a sua manipulação e uso continuo podem trazer algum risco a saúde. Pesquisas mais abrangentes e a longo prazo devem ser realizadas a fim de que duvidas como estas sejam sanadas, e tais produtos sejam inseridos ou não no mercado.

Palavras Chave: Nanocosmeticos, Envelhecimento, Pele.

Abstract: Aging is a natural process, which we are all susceptible. Today various technologies offer the treatment and consequent alleviation of their cutaneous signs. With the rise of cosmetic market consumption, also arise innovative techniques for various purposes and effective proposals. This article aims to review the literature on nanocosmetics and its performance in aging. For these purposes, several searches were done on the banks of scientific data, such as Medline, Pubmed, and Google Scholar. The Cosmetic market is increasing every year, and the investments in this segment as well. Nanotechnology has emerged as a major innovator for many aesthetic treatments, bringing promises of miraculous results. It is up to professionals in this area know and distinguish such products and their real capacity, harms and benefits. It is not known exactly which skin layers are affected if their effects are merely hydrants and if the risks to its handling and continued use may bring some risk to health. Extensive research over the long term and should be performed so that doubts as these are corrected, and such products are inserted or not on the market.

Keys-words: Nanocosmetics, Aging, Skin.

\section{I - INTRODUÇÃO}

A pele é grande reveladora do envelhecimento, pois é o órgão que através de sua aparência demonstra o avanço da idade sendo principalmente a 
face. Com isso, a qualidade da pele depende de uma série de fatores, tais como idade, sexo, clima, alimentação, hábitos e estado de saúde do indivíduo, podendo ser classificada em pele oleosa, mista e seca (SPIRDUSO, 2005).

O tema envelhecimento vem ganhando destaque em diferentes campos, em virtude do aumento da expectativa de vida, com consequente aumento da população em idade avançada, tornando os indivíduos preocupados com a aparência (MAIA 2008).

Atualmente, a medicina antienvelhecimento visa melhorar a qualidade de vida dos indivíduos durante este processo (JIMÉNEZ et al. 2006), uma vez que que este exerce influência direta nas atividades pessoais e profissionais dos mesmos (PACHECO, 2006). Diante desse tema, recentemente muito tem se discutido e estudado sobre vários produtos, dentre eles, os nanocosméticos, por ser dentro da estética e da cosmetologia um tratamento não invasivo e com resultados satisfatórios.

A nanotecnologia fundamenta-se na habilidade de caracterizar, manipular e organizar materiais em escala nanométrica. Trata-se de um campo científico multidisciplinar que se aplica a praticamente todos os setores da pesquisa, da engenharia de materiais e processos e de mercado (LEE, 2004). O princípio dessa nova ciência é que os materiais nesta escala nanométrica podem apresentar propriedades químicas, físico-químicas e comportamentais diferentes daquelas apresentadas em escalas maiores (WORLD NANOTECHNOLOGY MARKET, 2005). Portanto, este presente artigo objetiva revisar a literatura sobre nanocosméticos e sua atuação no envelhecimento.

\section{II - DESENVOLVIMENTO}

A pele é o maior órgão do corpo humano, reveste e delimita o organismo, corresponde $15 \%$ do peso corporal e tem por objetivo manter o meio interno em constante equilíbrio, protegendo e interagindo com o meio exterior, assim como os demais órgãos do corpo humano sofre alterações que caracterizam o envelhecimento cutâneo (AZULAY; AZULAY, 2006).

A ação que todos estão sujeitos e que compreende ao ciclo vital do organismo é o envelhecimento. Ocorre desde o nascimento, mas só aparecem 
sinais após a terceira idade, apresentando um ponto de vista amplo e variável de manifestações (GUIRRO \& GUIRRO, 2002). Envelhecer nada mais é que a diminuição geral das funções do organismo, e é um processo esperado, previsível, inevitável e progressivo (GOMES; GABRIEL, 2006).

A degeneração senil ocorre de preferência sobre regiões do tegumento que se acham expostas as intempéries, como por exemplo a face, pescoço, dorso das mãos e antebraços. A pele se pregueia, enruga, fica flácida e hiperpigmentada, provocando o agravamento ou exagero dos sulcos e pregas naturais das regiões comprometidas (HARRIS; HOFFMAN; CRUVINEL, 2005). Outro fator importante que contribui com o envelhecimento precoce da pele, é o excesso de expressão facial, isto é, algumas pessoas usam exageradamente e de forma errada alguns grupos musculares da face (GUIRRO \& GUIRRO, 2002).

Com o aumento da expectativa de vida e busca para prevenir e/ou retardar o aparecimento dos sinais de envelhecimento estimulam as pessoas a consumirem uma quantidade $\mathrm{e}$ variedade maior de produtos cosméticos por um período mais prolongado com a finalidade de manter a pele mais bonita, jovem e saudável (ABIHPEC, 2014).

Como consequência desses fatores, a indústria cosmética viu-se obrigada a repensar sua produção e desenvolver produtos cosméticos capazes de suprir as necessidades e exigências dos consumidores. Esta busca estimulou as empresas a investir em pesquisas de novos sistemas para incorporação de ativos cosméticos produzindo formulações mais eficazes e estáveis (MAGDASSI, 1997). As constantes pesquisas trouxeram avanços e inovações na área cosmética jamais vistas anteriormente. Essas pesquisas convergiram para uma tendência tecnológica mundial em todos os setores de produção: a nanotecnologia (WOKOVICH et al., 2009).

A nanotecnologia voltada para a cosmética tem como foco, sobretudo, os produtos destinados à aplicação na pele do rosto e do corpo, com ação antienvelhecimento e de fotoproteção, capazes de penetrar nas camadas mais profundas da pele, potencializando os efeitos do produto (NEVES, 2008). A técnica de fabricação de substâncias com dimensão menor que 100 nanômetros é chamado de 
nanotecnologia. $\mathrm{O}$ nanômetro corresponde à bilionésima parte do metro ou a milionésima parte do milímetro. A palavra nanotecnologia vem do grego, onde o prefixo nano significa "anão". (MARTINELLO, AZEVEDO, 2012). Dentre as quais as nanopartículas se destacam as poliméricas, subdivididas em nanocápsulas e nanoesferas, lipossomas, nanoemulsões, nanopartícula lipídica sólida entre outros (PAESE, 2008).

Entre os benefícios dos nanocosméticos destacam-se a melhor penetração de ingredientes ativos na pele e no cabelo, estabilização de substâncias, melhoria da eficácia e do sensorial dos produtos e a possibilidade de liberação controlada dos ativos (MARTINELLO, AZEVEDO, 2012). E algumas das vantagens podem ser obtidas com a utilização de nanocosméticos, como: proteção das matérias-primas quanto à degradação química ou enzimática, liberação gradual e em doses favoráveis (no caso de substâncias irritantes em altas doses), melhora na homogeneidade das formulações, aumento da estabilidade e da eficácia dos produtos e o aumento da capacidade de oclusão da pele, devido ao tamanho nanométrico dos sistemas (FRONZA et al., 2007; MÜLLER et al., 2002; SOMASUNDARAN et al., 2007; WEISS-ANGELI et al., 2008).

\section{Para GUIRRO \& GUIRRO} 2004, o processo fisiológico do envelhecimento torna $\mathrm{o}$ tecido conjuntivo gradualmente mais rígido, e diminui o número de fibras elásticas fazendo com que percamos a elasticidade natural da pele. $\mathrm{O}$ envelhecimento ocorre preferencialmente em regiões geralmente expostas ao sol ou regiões que excessivamente realizam mimicas, levando a degradação das fibras elásticas agravando sulcos e pregas das regiões comprometidas. Existem diversas teorias sobre o envelhecimento, dentre estas, a mais aceita aponta como responsável a ação dos Radicais Livres, onde o acumulo destes resulta em alterações oxidativas nas moléculas de longa vida, como colágeno, elastina e material cromossômico, levando a destruição de mucopolissacarídeos, acumulo de resíduos metabólicos inertes pela oxidação polimerizante, reações estas que envolvem lipídios, particularmente os poli-insaturados e proteínas, mudanças na integridade da 
membrana celular e fibrose arteriocapilar.

\section{III - DISCUSSÃO}

Sabendo que a nanotecnologia aplicada aos cosméticos tem como benefícios a melhora na permeação dos princípios ativos para ter sua propriedade efetivada, torna-se conveniente descrever o processo de permeação cutânea dos produtos bem como a diferenciação de locais e tempo de ação dos produtos com ou sem ativos nanoencapsulados (COSTA et al., 2004).

Vários estudos experimentais (NOHYNEK et al, 2007, 2008), investigaram a liberação na pele de nanoparticulas. A maioria desses trabalhos chegou a conclusões similares, no sentido de que as nanoparticulas penetram no estrato córneo, onde podem ser visualizadas, mas não penetram mais profundamente nas camadas viáveis da epiderme e da derme. Apenas alguns trabalhos sugeriram que a penetração na pele das nanoparticulas ocorre, mas, nesses casos, a penetração na pele observada poderia ser explicada pelos métodos experimentais ou de analises usados.
Além da pouca penetração comprovada, existem ainda riscos inerentes à saúde. Com a elevação do uso de nanoparticulas em cosméticos, aumentam também as precauções a serem tomadas. Estudos epidemiológicos mostram consistentemente que os aumentos das partículas atmosféricas provenientes do transporte terrestre $(60 \%)$ e dos processos de combustão (23\%) geraram aumentos, a curto prazo, na morbidez e na mortalidade via inalação (WIECHERS, 2009). Porém, de acordo com CARVALHO, 2010, as nanoparticulas podem oferecer novos riscos, embora a natureza desses possa ser grandemente hipotética.

A eficácia dos nanocosmeticos existentes no mercado, e sua proposta de regeneração de colágeno ainda é contestada. Tendo em vista que na pele envelhecida ocorreu a perda de elasticidade e demais componentes, a maneira possível de se reestabelecer sua produção seria a estimulação de um processo cicatricial. De acordo com ISAAC et al 2011, o processo cicatricial está divido em três fases onde ocorrem diversos processos fisiológicos que resultam na reformulação de fibroblastos e consequente colágeno. 
Para CAPEK 2004, as nanoemulsões, poderiam ser utilizadas como veículos para a liberação controlada e dispersão otimizada de ingredientes ativos para finalidades diversas como para tratamento de rugas. Já para NEVES 2008, quando se pretende um maior grau de penetração podem ser utilizados certos ativos para tais tratamentos. O que se observa é que a absorção nanocosmetica ainda não possui concordância quanto as camadas cutâneas atingidas e seus correspondentes resultados.

Ainda não existem estudos científicos que comprovem a ação nanocosmetica no envelhecimento, tendo em vista que para tal eficácia seriam necessários diversos processos fisiológicos que esta tecnologia ainda não comprovou ser capaz. São necessários estudos à longo prazo, para se descartar possíveis riscos e benefícios e alia-los ou não ao mercado cosmético.

\section{IV - CONSIDERAÕES FINAIS}

A nanotecnologia em cosméticos trouxe grandes avanços para o mercado da estética. Através dela foi possível preservar a matéria prima utilizada, (quanto

$\mathrm{a}$

degradação

química/enzimática), controlar a liberação de determinadas substancias que podem se tornar irritantes em altas doses e ainda possibilitou a melhora na homogeneidade destas formulações com consequente estabilidade cosmética.

Porém, os nanocosmeticos não apresentam eficácia comprovada no tratamento do envelhecimento facial, tendo em vista que não possuem ação inflamatória e/ou cicatricial para que haja reconstrução de colágeno. Se torna imprescindível mais pesquisas sobre o assunto para que se esclareça melhor seus benefícios e malefícios, riscos inerentes à exposição a longo prazo e ainda, a profundidade cutânea alcançada.

\section{V - REFERENCIAS}

ASSOCIAÇÃO BRASILEIRA DE INDUSTRIA DE HIGIENE PESSOAL, PERFUMARIA E COSMETICOS. (ABIHPEC). Panorama do setor Higiene Pessoal, Perfumaria e Cosméticos, 2014. Av. Paulista, 1313 cx 1080 - São Paulo - SP - Brasil email:comunicacao@abihpec.org.br. 
AZULAY, Rubem David; AZULAY, David Rubem. Dermatologia. 3.ed. Rio de Janeiro: Guanabara Koogan, 2006.

CAPEK I. Degradation of Kinetically stable $\mathrm{o} / \mathrm{w}$ emulsions. Advances in Colloid Interfacial Science, Amsterdam. v. 107, p. 125-55, 2004.

CARVALHO Dermeval de, Critical Reviews In Toxicology, 37:151$177,2007$.

CESAR Isaac, PEDRO Ribeiro Soares de Ladeira, FRANCCINI Mambrini Pires do Rêgo, JOHNY Conduta Borda Aldunate, RAFAEL Mamoru Carneiro Tutihashi, MARCUS Castro Ferreira. Alterações no processo de reparo fisiológico. Rev Bras Queimaduras. 2011;10(2):61-5.

COSTA A, KEDE M. P. V, SABATOVICH, O. Abordagem terapêutica tópica.. (ORGS). Dermatologia estética, 2. Ed., São Paulo: Atheneu, 2004. Cap. 4. 5. P. 8286.

FRONZA $\mathrm{T} ;$ GUTERRES $\mathrm{S}$; POHLMANN A; TEIXEIRA $\mathrm{H}$. Nanocosméticos: Em Direção ao
Estabelecimento de Marcos Regulatórios. Porto Alegre: UFRGS, 2007.

GIMÉNEZ JVG, GONZÁLEZ A, ALBANDEA N. Tratamiento del envejecimiento cutáneo mediante bioestimulación com factores de crescimiento autógenos. Rejuvenecimiento Cutáneo. 7(2): 8-14, 2005.

GOMES, R. K.; GABRIEL, M. Cosmetologia: descomplicando os princípios ativos. 2. ed. rev. e ampl. São Paulo: Livraria Médica Paulista, 2006.

GUIRRO, Elaine; GUIRRO, Rinaldo. Fisioterapia Dermato-funcional: fundamentos, recursos, patologias. 3.ed. São Paulo: Manole 2002.

GUIRRO, Elaine; GUIRRO, Rinaldo. Fisioterapia Dermato-funcional: fundamentos, recursos, patologias. 3.ed. São Paulo: Manole 2004.

HARRIS, M.; HOFFMANN, M.; CRUVINEL, A. Pele: estrutura, propriedades e envelhecimento. 2. ed. rev. e ampl. São Paulo: Senac, 2005.

ISAAC C, LADEIRA PRS, RÊGO 
FMP, ALDUNATE JCB, TUTIHASHI

RMC, FERREIRA MC. Alterações no processo de reparo fisiológico. Rev Bras Queimaduras. 2011;10(2):61-5.

JIMÉNEZ, Dr. J. Víctor García. GONZÁlEZ Dr. J. Antônio ALBANDEA, Nicolás. Tratamiento del envejecimiento cutâneo mediante BIOESTIMULACIÓN: Utilización de factores de CRECIMIENTO AUTÓGENOS (Parte II) Espanha Cortesía de Internacional Journal of Cosmetic Medicine and Surgery. Volumen 8 - Número 3 - 2006.

LEE, V. H. L. Nanotechnology: challenging the limit of creativity in targeted drug delivery. Advanced Drug Delivery Reviews, v. 56, p.1527-1528, 2004.

MAGDASSI, S. Delivery Systems in Cosmetics. Colloids and Surfaces v.123-124, p.671-679, 1997.

MAIA GF. Corpo e velhice na contemporaneidade. Estudos e pesquisas em psicologia UERJ. 8(3): 704-711, 2008.

MARTINELlO, Tiago. AZEVEDO, Valeska C. Nanotecnologia em
Cosmético. Instituto Racine, publicado em Indústria Farmacêutica.2012;

MÜLLER, $\quad$ RH.; $\quad$ RADTKE, $\quad$ M.; WISSING, SA. Solid lipid nanoparticles (SLN) and nanostructured lipid carriers (NLC) in cosmetic and dermatological preparations. Advanced Drug Delivery Reviews. v. 54, p.131-155, 2002.

NEVES, K. Nanotecnologia em cosméticos. Cosmetics \& Toiletries, v. 20, jan-fev, p.22, 2008.

NOHYNEK G J, ADEMANN J, RIBAUD C, ROBERTS M S. Grey goo on the skin? Nanotechnology, cosmetic and sunscreen safety, Crit Rev Toxicol 37:251-277, 2007.

NOHYNEK G J, DUFOUR $\mathrm{E} \mathrm{K}$, ROBERTS M S, Nanotechnology, cosmetic and the skin: is there a health risk? Skin Pharmacol Physiol 21: 136149, 2008.

PACHECO CA. A pele nossa de cada dia. Cosmetics \& Toiletries. 18: 28, 2006.

PAESE, K. Desenvolvimento tecnológico, estudo da fotoestabilidade e avaliação da permeação cutânea in 
vitro da benzofenona-3 a partir de nanocápsulas poliméricas incorporadas em diferentes veículos semi sólidos. Dissertação (Mestrado em Ciências Farmacêuticas) - Programa de PósGraduação em Ciências Farmacêuticas, UFRGS, 2008.

POHLMANN, AR.; GUTERRES, SS. Nanocapsules of octyl methoxycinnamate containing quercetin delayed the photodegradation of both components under ultraviolet A radition. Journal of Biomedical Nanotechnology. v.4. P1-10, 2008.

SPIRDUSO, WW. Dimensões Físicas do Envelhecimento. São Paulo: Manole, 2005.

SOMASUNDARAN, P.; MEHTA, SC.; RHEIN， L; CHAKRABORTY， S. Nanotechnology and related safety issuses for delivery of active ingredients in cosmetics. MRS Bulletin. v.32, p.779- 786, 2007.

WEISS-ANGELI, V. Desenvolvimento e caracterização de formulações fotoprotetoras contendo nanocápsulas. Tese (Doutorado em Ciências Farmacêuticas) - Programa de Pós -
Graduação em Ciências Farmacêuticas, UFRGS, 2007.

WIECHERS J W, Nanotechnology and skin delivery: Infinitely small or infinite possibilities? Cosm \& Toil 124(1):2839, 2009.

WOKOVICH, A.; TYNER, K.; DOUB, W.; SADRIEH, N.; BUHSE, LF. Particle size determination of sunscreens formulated with various forms of titanium dioxide. Drug Development and Industrial Pharmacy. p.1-10, 2009.

WORLD NANOTECHNOLOGY MARKET. An industry update. p.1-60, 2005.

WORLD NANOTECHNOLOGY MARKET. An industry update. RNCOS. p.1-60, 2005.

WEISS-ANGELI, V.; POLETTO, FS.; ZANCAN, LR.; BALDASSO, F.; 